\title{
Observatório Cidadão de Enchentes (OCE): uma plataforma de crowdsourcing para obtenção de VGI no contexto de gestão de risco de inundação
}

\author{
Lívia Castro Degrossi ${ }^{1}$, João Porto de Albuquerque ${ }^{1}$ \\ ${ }^{1}$ Instituto de Ciências Matemáticas e de Computação - Universidade de São Paulo (USP) \\ CEP 13566-590 - São Carlos - SP - Brazil \\ \{degrossi, jporto\}eicmc.usp.br
}

\begin{abstract}
The number and intensity of floods have increased worldwide due to climate change, causing more damage, deaths and economic impacts than any other natural phenomenon. Prevent this type of disaster requires current, complete and accurate information on the current state of the environmental variable. Crowdsourcing platforms enable obtaining volunteered geographic information for different contexts. We propose a crowdsourcing platform for obtaining useful volunteer information for the context of flood risk management, an experimental evaluation is performed in order to verify its effectiveness.
\end{abstract}

Resumo. O número e a intensidade das inundações têm aumentado em todo o mundo devido às mudanças climáticas, causando mais danos, mortes e impactos econômicos do que qualquer outro tipo de fenômeno natural. Prevenir esse tipo de desastre requer informações atualizadas, completas e precisas sobre o atual estado das variáveis ambientais. As plataformas de crowdsourcing possibilitam a obtenção de informações geográficas voluntárias para diferentes contextos. Nesse contexto, é proposta uma plataforma de crowdsourcing para a obtenção de informações voluntárias úteis para o contexto de gestão de risco de inundação, sendo realizada uma avaliação experimental, a fim de verificar sua eficácia.

\section{Introdução}

As alterações climáticas estão aumentando o número e a intensidade de desastres naturais em todo o mundo [UN 2013]. Entre 2000 e 2012, os desastres naturais provocaram aproximadamente U\$ 1,7 trilhão em prejuízos econômicos, além de afetar 2,9 bilhões de pessoas e provocar a morte de 1,2 milhão [UNISDR 2013]. A ocorrência de desastres naturais está ligada não somente às características geoambientais, mas também à vulnerabilidade do sistema social [Alcántara-Ayala 2002]. Nessa perspectiva, a gestão de desastres (GD) visa reduzir ou evitar potenciais perdas e garantir assistência às vítimas. Em particular, informações espaciais têm sido fundamentais para todo o processo de GD [Tu et al. 2009]. Além disso, esse tipo de informação é essencial em todas as fases do processo para que ações sejam realizadas a fim de reduzir os impactos provocados ou identificar sua iminência. Para isso, as informações devem ser confiáveis, precisas e atualizadas [Poser and Dransch 2010, Tu et al. 2009].

Em particular, as inundações representam cerca de $30 \%$ dos desastres naturais que ocorrem em todo o mundo, causando mais danos, mortes e impactos econômicos 
do que qualquer outro tipo de evento [van der Kooij 2013]. No Brasil, os problemas com inundações se intensificam no período de chuvas, que compreende os meses de dezembro a março. A gestão de risco de inundação (GRI) consiste no processo de gerenciar uma situação de risco de inundação existente, controlando os impactos provocados pela inundação, estando preparado para ela [Plate 2002]. Para isso, são necessárias informações atualizadas sobre o estado das variáveis ambientais, as quais permitem a simulação dos efeitos e da severidade de um desastre [van der Kooij 2013, Plate 2002]. Esse tipo de simulação auxilia a reduzir o impacto das inundações, ao permitir que a população das áreas afetadas tomem medidas preventivas, além de permitir que agências de emergência desenvolvam planos de resposta.

Informações espaciais coletadas por voluntários, chamadas Volunteered Geographic Information (VGI) [Goodchild 2007], estão sendo utilizadas como fonte de dados para a gestão de desastres. Isso se deve ao fato de que VGI oferece um número potencialmente grande de voluntários que atuam como "sensores", observando importantes parâmetros locais para a gestão de desastres [Poser and Dransch 2010]. Em particular, as plataformas de crowdsourcing existentes possibilitam a coleta de informações voluntárias referentes à fase de resposta, como número de vítimas, pontos de distribuição de alimentos, etc. Ainda, não há uma plataforma de crowdsourcing configurada para a obtenção de informações voluntárias úteis para a fase de preparação no contexto de gestão de risco de inundação. Além disso, o monitoramento contínuo do risco de inundação requer informações locais específicas, como precipitação e altura da água, para avaliar o potencial risco de inundação. Assim, os autores deste trabalho propõem a criação de uma plataforma de crowdsourcing, a partir da configuração de uma plataforma existente, para a obtenção de informações geográficas voluntárias úteis para o contexto de gestão de risco de inundação.

O restante deste artigo está estruturado da seguinte forma: a Seção 2 apresenta a fundamentação teórica deste trabalho. Na Seção 3 é apresenta a plataforma de crowdsourcing proposta neste trabalho. A Seção 4 apresenta a avaliação experimental conduzida como forma de avaliação da plataforma. Na Seção 5 são apresentados e discutidos os resultados da avaliação experimental realizada. Por fim, na Seção 6 são apresentadas as conclusões e trabalhos futuros.

\section{Fundamentação Teórica}

\subsection{Gestão de Risco de Inundação}

A gestão de risco de inundação (GRI) é o processo de gerenciar uma situação de risco de inundação existente. Seu objetivo é controlar uma inundação, estando preparado para ela e minimizando seus impactos [Plate 2002]. Dentre os desastres naturais que ocorrem em todo o mundo, as inundações são as mais frequentes. O crescente aumento no número de inundações está relacionado às mudanças climáticas, sendo agravada pelo crescimento urbano desordenado e pelo fenômeno da urbanização acelerada sem a disponibilidade dos serviços essenciais [da Silva 2009, MIN 2013].

$\mathrm{Na}$ GRI, a fase de preparação possui como objetivo principal reduzir o risco residual por meio de sistemas de alerta e medidas que podem ser realizadas a fim de minimizar os impactos provocados por uma inundação. Para isso, é necessário o monitoramento constante dos riscos existentes e uma avaliação do perigo decorrente a partir de 
informações recentes.

Atualmente, informações geográficas e tecnologias relacionadas possuem papel fundamental em todas as fases da GRI. Desastres naturais são tipicamente monitorados utilizando diferentes dispositivos, como sensores, satélites, sismógrafos, entre outros. Contudo, esses dispositivos não oferecem informações sobre os impactos provocados por tais desastres.

\subsection{Volunteered Geographic Information}

Com o avanço da Internet e dos dispositivos móveis, os usuários passaram não só a utilizar as informações geográficas disponíveis online, mas também a fornecê-las. Esse tipo de informação é denominada Volunteered Geographic Information (VGI) [Goodchild 2007]. Outrora, a criação de informações geográficas era realizada somente por agências oficiais. Contudo, com o aumento das interações viabilizadas pela Web 2.0, o uso de dispositivos com GPS (Global Positioning System) e o acesso a Internet com conexão de alta capacidade, as informações geográficas estão sendo produzidas por cidadãos que possuem pouca qualificação formal. Entre as vantagens associadas ao VGI, pesquisadores destacam seu uso para enriquecer, atualizar ou completar conjuntos de dados geoespaciais existentes [Goodchild 2007].

Um certo número de aplicações desfrutam do uso de VGI como fonte de informação. Um dos exemplos mais interessantes do uso de VGI é a plataforma Wikimapia ${ }^{1}$. Essa é uma plataforma de mapeamento colaborativo que tem como objetivo marcar e descrever objetos geográficos em todo o mundo. De maneira semelhante, a plataforma colaborativa OpenStreetMap ${ }^{2}$ visa criar um mapa de todo o mundo a partir de dados fornecidos por cidadãos. A origem dessa plataforma foi inspirada no sucesso de outra plataforma colaborativa, a Wikipédia ${ }^{3}$. Essa é uma enciclopédia colaborativa e multilíngue, onde o conteúdo é escrito colaborativamente por voluntários ao redor do mundo.

Desastres naturais recentes têm mostrado que informações de voluntários, fornecidas pela Internet, podem melhorar a consciência situacional, fornecendo uma visão geral sobre a presente situação [Poser and Dransch 2010]. Tal fato ocorre, pois VGI oferece uma grande oportunidade para aumentar a consciência devido ao número potencialmente grande de voluntários, mais de seis bilhões de pessoas, que agem como "sensores", observando importantes parâmetros da gestão de desastres em um ambiente local [Goodchild 2007, Poser and Dransch 2010, Schade et al. 2013]. Ainda, apesar dos recentes avanços no desenvolvimento de sensores, suas observações podem não estar disponíveis devido a interrupções de comunicação ou, até mesmo, a destruição do sensor, além de os mesmos não serem capazes de medir certos fenômenos, como tempestades de granizo [Poser and Dransch 2010]. [Poser and Dransch 2010] utilizaram informações geográficas provindas de cidadãos afetados pela inundação para estimar os impactos por ela causados.

Contudo, apesar das vantagens da participação da população em coletar informações, há um grande número de desafios a serem enfrentados. Apesar do potencial

\footnotetext{
${ }^{1}$ http://www.wikimapia.org

${ }^{2}$ http://www.openstreetmap.org

${ }^{3}$ http://www.wikipedia.org
} 
do VGI, a qualidade dos dados é a maior preocupação. Informações provenientes de muitos indivíduos pode ocasionar dúvidas sobre a credibilidade [Erskine and Gregg 2012]. Além disso, não se sabe de antemão quanto e a partir de onde a informação será fornecida. Outro desafio enfrentado refere-se à localização, diferentemente dos sensores in-situ, as pessoas estão em constante movimento, assim as observações por elas realizadas precisam ser localizadas para que se tornem úteis [Poser and Dransch 2010]. Além disso, VGI é, muitas vezes, considerada como insuficientemente estruturada, documentada e validada [Longueville et al. 2010b].

Nesse cenário, diferentes plataformas de software vêm sendo empregadas com o intuito de coletar informações geográficas voluntárias, permitir sua visualização e análise. Dentre as plataformas existentes, a plataforma Ushahidi tem sido usada em resposta a dezenas de desastres naturais em todo o mundo [Meier 2013].

\subsection{Observatório Cidadão}

Atualmente, diversas plataformas de crowdsourcing apoiam a gestão de desastres, permitindo coletar informações de cidadãos das áreas atingidas, bem como sua análise e visualização. Ao utilizar voluntários para desempenhar uma tarefa específica, como o monitoramento ambiental, esses se tornam coletivamente um Observatório Cidadão, onde os dados podem ser coletados, coligidos e publicados [Miorandi et al. 2013]. Assim, o termo Observatório Cidadão (OC) pode ser entendido como uma plataforma de software a fim de obter informações voluntárias sobre um tema específico, por meio de diferentes dispositivos (navegador de um computador, aplicativo para celular e SMS), além de permitir sua visualização. Esse, por sua vez, pode ser utilizado para compartilhar informações sobre os riscos relacionados à inundação, como altura da água no leito do rio ou áreas alagadas.

Um OC foi utilizado durante o terremoto que atingiu o Haiti em 2010 [Zook et al. 2010]. Nele os cidadãos das áreas atingidas podiam enviar relatos sobre incidentes ocorridos nessas áreas. Nesse mesmo ano, um terremoto atingiu o Chile, acarretando em milhares de vítimas. Inicialmente foram coletadas informações da população local, por meio de mensagens de texto e de fontes de mídias tradicionais [Carlsen et al. 2011]. Essas informações foram, então, mapeadas para um OC, apoiando as agências de emergência e órgãos governamentais. Em 2011, durante as inundações ocorridas em Queensland, a Australian Broadcasting Corporation lançou um OC para mapear as áreas inundadas [McDougall 2011]. Esse permitiu que indivíduos enviassem informações sobre a inundação por meio de e-mail, mensagens de texto, Twitter ou pela própria plataforma, sendo essas informações disponibilizadas para qualquer pessoa que tivesse acesso à internet. Ainda, outros OC foram empregados durante eventos extremos, como durante as inundações no Paquistão em $2010^{4}$, Boulder (USA) em $2013^{5}$, entre outros. Contudo, os observatórios cidadãos existentes estão configurados para a obtenção de informações voluntárias referentes aos impactos provocados por um desastre, como número de vítimas, etc. Dessa forma, não há um observatório cidadão configurado para a obtenção de informações voluntárias refente à fase de preparação da gestão de risco de inundação que satisfaça as necessidades informacionais do Brasil.

\footnotetext{
${ }^{4}$ http://pakreport.org/ushahidi/

${ }^{5}$ https://boulderflood2013b.crowdmap.com/
} 


\section{Observatório Cidadão de Enchentes}

O Observatório Cidadão de Enchentes (OCE) (Figura 1) consiste em uma plataforma de crowdsourcing que possibilita a obtenção de informações voluntárias. Seu principal objetivo é coletar informações geográficas voluntárias úteis para o contexto de gestão de risco de inundação, mais especificamente sobre áreas alagadas e altura da água no leito do rio. Para isso, os voluntários podem enviar informações de diferentes dispositivos, como o aplicativo para celular ou pelo Web site. Para tanto, o OCE consiste em uma instância da plataforma de crowdsourcing Ushahidi, pois essa é mundialmente utilizada por ativistas, agências de emergência e cidadãos para mapear eventos extremos [Ushahidi 2014].

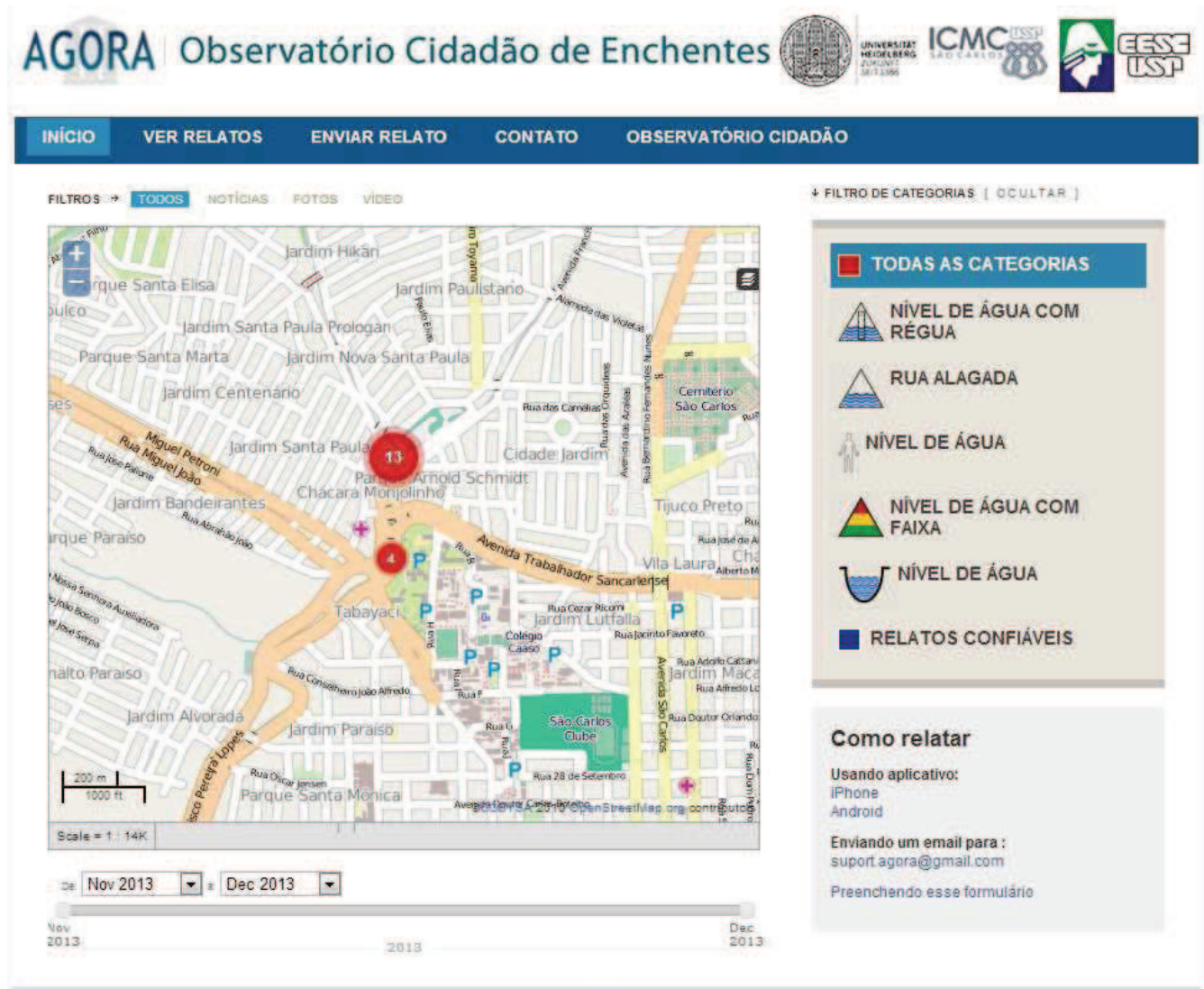

Figura 1. Observatório Cidadão de Enchentes.

A fim de facilitar o fornecimento de informações sobre o risco de inundação no OCE, foram definidos mecanismos, realizado em conjunto com pesquisadores da área de hidrologia, para apoiar os voluntários a melhor interpretar a altura da água no leito do rio. Para tanto, três diferentes cenários foram considerados a fim de que o voluntário possa interpretar as variáveis ambientais em qualquer situação. O primeiro cenário corresponde a um ponto controlado, ou seja, há um recurso de interpretação que possibilita realizar a medição de forma mais precisa. Nesse cenário há uma régua linimétrica fixada no leito do rio que apoia a medição da altura da água do mesmo. O segundo cenário corresponde a um ponto semicontrolado, ou seja, um ponto onde há um recurso de interpretação que permite determinar o valor da altura da água no leito do rio de forma menos precisa. Nesse cenário há dois tipos de recursos para interpretação, um boneco similar à figura humana e faixas de cores (verde, amarelo, laranja e vermelho). As faixas de cores correspondem ao índice 
de perigo (IP), isto é, o perigo ao qual a população está exposta, o risco de instabilidade humana. Esse risco representa as forças exercidas sobre um indivíduo em correntes de água, ou seja, o nível de vulnerabilidade desse exposto a enchentes [Rotava et al. 2013]. O boneco foi proposto, pois auxilia os voluntários a determinar a altura da água com maior facilidade, sendo essa realizada de acordo com marcações pré-definidas, isto é, tornozelo, joelho, cintura, pescoço e acima da cabeça (corpo total). Por fim, o terceiro cenário corresponde a um ponto não controlado, isto é, não há nenhum recurso no leito do rio que auxilie os voluntários a interpretar a altura da água. Para esse mecanismo, são adotadas marcações (baixo, normal, alto e transbordando) que se aproximam do conhecimento popular e que são de simples determinação. Os três primeiros recursos podem ser observados na Figura 2.

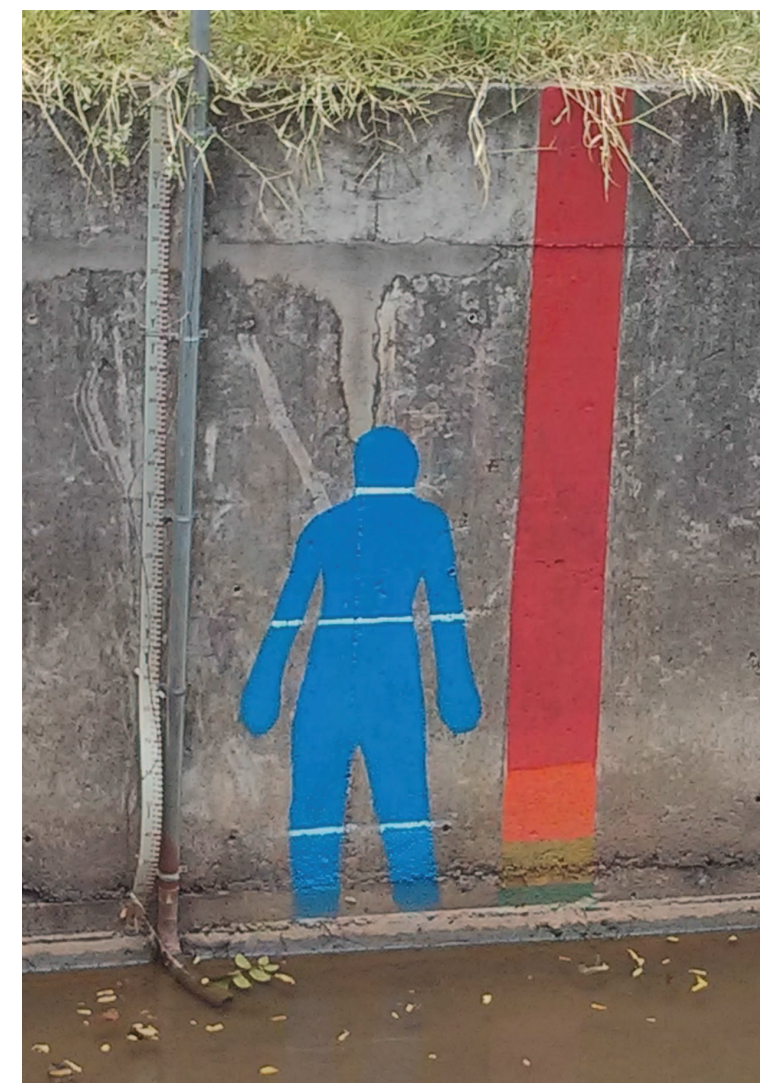

Figura 2. Recursos para determinação da altura da água no leito do rio.

No OCE, cada mecanismo é representado por meio de uma categoria distinta, sendo as marcações desses representadas por subcategorias. Dessa forma, o voluntário pode identificar mais facilmente a categoria que melhor representa o cenário observado.

Para enviar um relato, os voluntários podem utilizar tanto o aplicativo para celular quanto o Web site. O envio de um relato requer que o voluntário forneça algumas informações obrigatórias, sendo as demais informações opcionais (nome, sobrenome, email, link de fonte de notícias, link de Vídeo Externo e fotos):

- Título: representa o assunto abordado no relato;

- Descrição: representa a observação realizada pelo voluntário, por exemplo, a altura da água ou área alagada; 
- Categoria: representa o mecanismo utilizado para interpretação da variável ambiental, o qual fornece informações sobre a altura da água no leito do rio e áreas alagadas;

- Nome do local: representa o local de onde o voluntário está enviando o relato.

Vale ressaltar que no caso do envio pelo Web site, é de responsabilidade do voluntário assinalar no mapa o ponto ao qual o relato está se referindo. Assim, é possível relacionar o incidente reportado a um local específico. Por outro lado, no caso do aplicativo para celular, uma vez disponível do GPS do mesmo, a posição geográfica é definida de forma automática.

Em virtude da incerteza acerca da credibilidade dessas informações, essas são verificadas antes de serem disponibilizadas para a visualização. O objetivo dessa verificação é reduzir o número de informações falsas ou imprecisas. Assim, antes dos relatos serem disponibilizados online, o administrador da plataforma deve aprová-los. Uma vez aprovado, esse pode ter dois status distintos: verificado e não verificado. O status "verificado" indica que a informação presente no relato pode ser confirmada por outras fontes externas, como diferentes mídias e outros voluntários. Em contrapartida, o status "não verificado" indica que não ocorreu nenhuma verificação relacionada ao incidente reportado, ou seja, o administrador não encontrou outras notícias na mídia que confirmassem tal acontecimento ou nenhum outro voluntário reportou o mesmo incidente. Inicialmente, os autores desse trabalho exercem o papel de administrador da plataforma, aprovando e verificando os relatos inseridos na mesma. Contudo, espera-se que agências de emergência, envolvidas na GRI, desempenhem futuramente esse papel.

\section{Avaliação Experimental}

Como forma de avaliação da plataforma Observatório Cidadão de Enchentes, um experimento foi realizado com o objetivo de verificar a obtenção de informações voluntárias úteis para o contexto de gestão de risco de inundação. Em particular, o experimento buscou verificar se essa plataforma é eficaz para a obtenção de informações voluntárias úteis e se a diferença entre a média dos valores contidos nessas informações e a média dos dados de sensores é significativa. Assim, para alcançar tais objetivos, duas questões foram formuladas pelos autores desse trabalho, a fim de direcionar sua execução. A primeira questão faz referência à eficácia da plataforma em obter informações voluntárias para o contexto de gestão de risco de inundação. Dessa forma, essa questão foi definida como:

Q1) A plataforma de crowdsourcing, Observatório Cidadão de Enchentes, é eficaz para a obtenção de informações voluntárias úteis para o contexto de gestão de risco de inundação?

A segunda questão foi formulada a fim de verificar se a diferença entre a média das informações voluntárias e a média dos dados de sensores é significativa, pois caso haja uma diferença significativa, essas podem camuflar o real estado da variável ambiental, acarretando em previsões errôneas do risco de inundação. Assim, a seguinte questão foi elaborada:

Q2) A diferença entre a média das informações voluntárias e a média dos dados de sensor é significativa? 
Uma vez formuladas, para cada questão foi definida uma hipótese nula, a qual se deseja refutar com maior significância possível, e uma hipótese alternativa, a qual se pretende comprovar. Em relação à eficácia da plataforma de crowdsourcing, levantada pela Q1, procurou-se refutar a afirmação de que a porcentagem de informações voluntárias úteis obtidas pela plataforma é menor ou igual a 50\% (Hipótese Nula). Por outro lado, buscou-se comprovar que a porcentagem de informações voluntárias úteis obtidas pela plataforma é maior que 50\% (Hipótese Alternativa). Uma informação voluntária é considerada útil se essa pode ser utilizada por modelos hidrológicos ou para a tomada de decisão por agências de emergência. Dessa forma, é possível assegurar que a plataforma proposta é eficaz se a porcentagem de informações voluntárias úteis por ela obtidas for elevada. Em relação à diferença entre a média das informações voluntárias e a média dos dados de sensor, levantada pela Q2, procurou-se refutar a afirmação de que a média dos valores contidos nas informações voluntárias é diferente da média dos valores medidos pelo sensor (Hipótese Nula). Por outro lado, buscou-se comprovar que a média dos valores contidos nas informações voluntárias é igual à média dos valores medidos pelo sensor (Hipótese Alternativa).

Durante a fase de planejamento do experimento, duas variáveis dependentes e uma independentes foram definidas, as quais estão envolvidas no contexto dessa abordagem. Após a definição das variáveis do experimento, métricas foram elaboradas para medir essas variáveis. No contexto da plataforma, uma informação voluntária é considerada útil se a categoria selecionada pelo voluntário representar o cenário por ele observado. Dessa forma, um critério foi definido a fim de determinar a utilidade dessa informação para o contexto de gestão de risco de inundação. Para tanto, três valores distintos foram atribuídos ao critério: zero (não representa o cenário observado, ou seja, a categoria selecionada não condiz com a descrição do relato), cinco (representa parcialmente o cenário observado, isto é, o voluntário selecionou mais de uma categoria), dez (representa completamente o cenário observado, ou seja, o voluntário selecionou apenas uma categoria que representa exatamente o cenário observado). Assim, a eficácia da plataforma é calculada pela porcentagem de informações voluntárias que possuem nota maior ou igual a cinco nesse critério. Ainda, a média dos valores obtidos nas informações voluntárias é calculada pela soma dos valores contidos na descrição do relato, referente à altura da água na régua, e dividida pelo número de participantes do experimento. Por outro lado, a média dos valores medidos pelo sensor é calculada pela soma das medições realizadas pelo sensor, no período de tempo em que os voluntários realizaram as observações, e dividida pelo número de medições realizadas nesse período.

Para a fase de execução do experimento, foram levados em consideração um fator, altura da água, e dois tratamentos, altura da água observada pelos voluntários e altura da água medida pelo sensor. Além disso, para a execução do experimento, os participantes foram selecionados de forma estratégica, visando selecionar participantes com diferentes níveis de experiência e conhecimento na área de gestão de risco de inundação. Ainda, antes da realização do experimento, os participantes passaram por um treinamento que possibilitou apresentar a plataforma de crowdsourcing, proposta nesse trabalho, e enunciar os mecanismos utilizados para a interpretação da altura da água no leito do rio.

Para a fase de análise e interpretação dos resultados, foram utilizados três testes estatísticos. Primeiramente, o Teste de Shapiro-Wilk [Royston 1983] foi utilizado para 
verificar a normalidade das amostras. Em seguida, o Teste de Levene [Olkin 1960] foi aplicado para verificar a homocedasticidade da amostra, ou seja, se as diferentes amostras apresentam igualdade de variância. Por fim, o Teste T [Wohlin et al. 2000] foi aplicado a fim de refutar ou aceitar estatisticamente a hipótese nula. Segundo [Travassos 2011], é possível rejeitar a hipótese nula com base nos resultado do teste estatístico.

\section{Resultado e Discussão}

Anteriormente à execução do experimento, foi conduzido um estudo piloto, com nove participantes, para avaliar o protocolo experimental. Em seguida, um experimento foi realizado com dez voluntários (participantes), com diferentes níveis de experiência e conhecimento na área de gestão de risco de inundação (Tabela 1), em um ponto da bacia hidrográfica da cidade de São Carlos/SP. Durante a execução, os voluntários inseriram quinze relatos na plataforma.

\section{Tabela 1. Experiência dos participantes no contexto de gestão de risco de inundação obtida por meio do questionário.}

\begin{tabular}{|c|c|}
\hline ID do participante & Tempo de Experiência \\
\hline 10 & 0 mês \\
\hline 11 & 0 mês \\
\hline 12 & 1 mês \\
\hline 13 & 0 mês \\
\hline 14 & 0 mês \\
\hline 15 & 0 mês \\
\hline 16 & 7 meses \\
\hline 17 & 240 meses \\
\hline 18 & 12 meses \\
\hline 19 & 12 meses \\
\hline
\end{tabular}

Primeiramente, os relatos foram analisados quanto à categoria selecionada pelo voluntário, a fim de verificar a eficácia da plataforma. Para cada um dos relatos foi atribuído uma nota de acordo com o número de categorias selecionadas e se essas representavam completamente ou parcialmente o cenário observado ou, ainda, não o representavam, de acordo com a descrição do relato. Dentre os relatos inseridos, nove possuíam apenas uma categoria selecionada, a qual representava completamente o cenário observado. A esses foi atribuído o valor 10. Dois relatos possuíam mais de uma categoria selecionada, as quais representavam parcialmente o cenário observado. Para cada relato foi atribuído o valor 5. Por fim, quatro relatos possuíam uma ou mais categorias selecionadas, porém essas não representavam o cenário observado. A esses foi atribuído o valor 0 . Assim, onze relatos obtiveram valor igual ou maior do que cinco, ou seja, aproximadamente $73 \%$ dos relatos inseridos podem ser utilizados para o contexto de gestão de risco de inundação. Dessa forma, é possível concluir que por meio das categorias da plataforma o voluntário consegue enviar informações úteis para esse contexto. Desse modo, a hipótese nula foi refutada, ou seja, a plataforma é eficaz para a obtenção de informações voluntárias úteis para o contexto de gestão de risco de inundação. Esse resultado pode ser justificado pelo fato de que todos os voluntários do experimento passaram por um treinamento antes da sua execução, auxiliando-os a determinar a altura da água com maior 
facilidade, além de inserir suas observações na plataforma mais facilmente. Dessa forma, é possível afirmar que o treinamento foi comprovadamente suficiente para que os voluntários produzissem observações úteis.

Em seguida, os relatos relacionados à altura da água na régua linimétrica foram analisados, a fim de verificar se a diferença entre a média desses valores e média dos dados do sensor era significativa. Para tanto, apenas dez relatos foram analisados, visto que somente esses possuíam o valor da altura da água na régua linimétrica. Primeiramente foi realizada uma correspondência entre os valores observados pelos voluntários e os valores medidos pelo sensor. Essa etapa foi realizada pois a régua linimétrica se encontrava mal fixada junto ao leito do rio. Em seguida, foi realizado o Teste de Shapiro-Wilk para averiguar se as amostras possuíam distribuição normal. A partir dos resultados obtidos (Tabela 2) foi possível constatar que a média dos valores contidos nas informações voluntárias não possui distribuição normal, pois o valor de Sig. (p-valor) é menor que o nível de significância ${ }^{6}$ adotado (5\%). Em contrapartida, a média dos valores medidos pelo sensor possui distribuição normal, pois o valor de Sig. é maior que o nível de significância.

Tabela 2. Resultado do Teste de Shapiro Wilk.

\begin{tabular}{|c|c|c|}
\hline \multirow{2}{*}{ Variável Testada } & \multirow{2}{*}{ Instrumento } & Teste de Shapiro Wilk \\
\cline { 3 - 3 } & & Sig. \\
\hline \multirow{2}{*}{ Altura da Água } & Voluntário & 0,004 \\
\cline { 2 - 3 } & Sensor & 0,494 \\
\hline
\end{tabular}

Para amostras com distribuição normal, o teste de hipótese mais adequado é o Teste T. Segundo [Wohlin et al. 2000], esse teste pode, também, ser aplicado para amostras que não possuem distribuição normal desde que essas não contenham muitos elementos. Assim, esse teste foi selecionado para verificar se a diferença entre a média dos valores contidos nas informações voluntárias e a média dos valores medidos pelo sensor era significativa.

Após a realização do Teste de Levene, ao verificar o valor obtido de Sig. (p-valor) (Tabela 3), foi possível constatar que as amostras não possuem igualdade de variância, visto que esse valor é menor que o nível de significância. Assim, ao realizar o Teste T, o valor analisado corresponde a amostras que não possuem igualdade de variância. Em seguida, o Teste $\mathrm{T}$ foi realizado para verificar se a diferença entre as médias era significativa. O resultado obtido (Tabela 3 ) comprova que a diferença entre as médias não é estatisticamente significativa, pois o valor de Sig (2-tailed) é maior que o nível de significância. Esse resultado pode ser confirmado ao observar a diferença entre as médias, apresentada na Tabela 3. Assim, foi possível rejeitar a hipótese nula, visto que o valor de p-valor (Sig. (2-tailed)) é maior que o nível de significância. Vale ressaltar que esse resultado pode ter sido ameaçado, pois a régua linimétrica não estava bem fixada à margem do leito do rio (Figura 2), o que pode ter ocasionado uma interpretação errônea da altura da água pelos voluntários.

\footnotetext{
${ }^{6} \mathrm{O}$ nível de significância corresponde a probabilidade de rejeitar a hipótese nula sendo a mesma verdadeira.
} 
Tabela 3. Resultado do Teste de Levene e do Teste T.

\begin{tabular}{|c|c|c|c|c|c|}
\hline \multirow{2}{*}{ Variável Testada } & \multirow{2}{*}{ Instrumento } & \multirow{2}{*}{ Média } & Teste de Levene & \multicolumn{2}{|c|}{ Teste T } \\
\cline { 3 - 6 } & & Sig. & $\begin{array}{l}\text { Sig. (2- } \\
\text { tailed) }\end{array}$ & $\begin{array}{l}\text { Diferença } \\
\text { Média }\end{array}$ \\
\hline \multirow{2}{*}{ Altura da Água } & Voluntário & 21,13 & \multirow{2}{*}{0,002} & 0,093 & $-2,799$ \\
\cline { 3 - 3 } & Sensor & 23,93 & & \\
\hline
\end{tabular}

\section{Conclusão e Trabalhos Futuros}

Com base no experimento realizado, foi possível constatar que a plataforma de crowdsourcing, Observatório Cidadão de Enchentes, é eficaz para a obtenção de informações voluntárias úteis para o contexto de gestão de risco de inundação. Isso ocorre, pois os voluntários puderam representar o estado das variáveis ambientais (altura da água e área alagada), no cenário por eles observado, por meio da seleção da categoria mais adequada. Além disso, foi possível verificar que não existe uma diferença estatisticamente significativa entre a média dos valores contidos nas informações voluntárias e a média dos valores medidos pelo sensor. Assim, a hipótese nula pode ser refutada. Contudo, os resultados podem ter sido alterados, pois a régua linimétrica não estava bem fixada junto ao leito do rio.

Com relação às possibilidades de trabalhos futuros, é necessário conduzir outro experimento em diferentes pontos da bacia hidrográfica de São Carlos/SP, a fim de comprovar que a diferença entre a média dos valores observados pelos voluntários e a média do valores medidos pelos sensores não é estatisticamente significativa. Além disso, é necessário realizar um experimento com voluntários não treinados para verificar se os mecanismos propostos os apoiam a determinar a altura da água e se é possível enviar uma observação, por meio da plataforma, com facilidade e de maneira eficaz.

\section{Referências}

Alcántara-Ayala, I. (2002). Geomorphology, natural hazards, vulnerability and prevention of natural disasters in developing countries. Geomorphology, 47:107-124.

Carlsen, J., Dougherty, J., Iacucci, A. A., Rodriguez, D., Sonoyama, S., and Stauffer, C. (2011). Grant report. Technical report, Mozilla Corporation.

da Silva, J. P. R. P. (2009). Mapeamento de inundações no brasil: proposta de gestão ambiental através de um sistema de informações geográficas. In VI Congresso de Meio Ambiente da AUGM.

Erskine, M. A. and Gregg, D. G. (2012). Utilizing volunteered geographic information to develop a real-time disaster mapping tool: A prototype and research framework. In International Conference on Information Resources Management.

Goodchild, M. F. (2007). Citizens as sensors: the world of volunteered geography. GeoJournal, 69(4):211-221.

Longueville, B. D., Annoni, A., Schade, S., Ostlaender, N., and Whitmore, C. (2010a). Digital earth's nervous system for crisis events: real-time sensor web enablement of volunteered geographic information. International Journal of Digital Earth, 3(3):242259. 
Longueville, B. D., Luraschi, G., Smits, P., Peedell, S., and Groeve, T. D. (2010b). Citizens as sensors for natural hazards: A vgi intergration workflow. Geomatica, 64:41-59.

McDougall, K. (2011). Using volunteered information to map the queensland floods. In Proceedings of the Surveying \& Spatial Sciences Biennal Conference.

Meier, P. (2013). Crisis maps: Harnessing the power of big data to deliver humanitarian assistance. Acessado em 27/12/2013.

MIN, M. D. I. N. (2013). Defesa civil - apresentação. Acessado em: 21/12/2013.

Miorandi, D., Carreras, I., Gregori, E., Graham, I., and Stewart, J. (2013). Neutrality in mobile internet: Towards a crowdsensing-based citizen observatory. In IEEE International Conference on Communications.

Olkin, I. (1960). Contributions to Probability and Statistics: Essays in Honor of Harold Hotelling. Stanford University Press.

Plate, E. J. (2002). Flood risk and flood management. Journal of Hydrology, 267:2-11.

Poser, K. and Dransch, D. (2010). Volunteered geographic information for disaster management with application to rapid flood damage estimation. Geomatica, 64(1):89-98.

Rotava, J., Mendiondo, E. M., and Souza, V. C. B. (2013). Simulação de instabilidade humana em inundações: Primeiras considerações. In XX Simpósio Brasileiro de Recusos Hídricos.

Royston, J. P. (1983). Some techiniques for assessing multivarate normality based on the shapiro-wilk w. Journal of the Royal Statistical Society, 32:121-133.

Schade, S., Díaz, L., Ostermann, F., Spinsanti, L., Luraschi, G., Cox, S., Nuñez, M., and Longueville, B. D. (2013). Citizen-based sensing of crisis events: sensor web enablement for volunteered geographic information. Applied Geomatics, 5(1):3-18.

Travassos, G. H. (2011). Experimentação em engenharia de software: Fundamentos e conceitos. In VIII Experimental Software Engineering Latin American Network.

Tu, Y., Li, Q., and Liu, R. (2009). A geospatial information portal for emergency management of natural disasters. In IEEE International Geoscience and Remote Sensing Symposium, volume 2, pages II-404 - II-407.

UN, U. N. (2013). Humanitarian and disaster relief assistance. Acessado em: 02/12/2013.

UNISDR, U. N. O. F. D. R. R. (2013). Economic losses from disasters set new records in 2012. Acessado em: 02/12/2013.

Ushahidi (2014). The ushahidi platform. Acessado em: 10/01/2014.

van der Kooij, M. (2013). Flood monitoring and disaster management response. GEOconnexion International Magazine, pages 26-28.

Wohlin, C., Runeson, P., Höst, M., Ohlsson, M. C., Regnell, B., and Wesslén, A. (2000). Experimentation in software engineering: an introduction. Kluwer Academic Publishers.

Zook, M., Graham, M., Shelton, T., and Gorman, S. (2010). Volunteered geographic information and crowdsourcing disaster relief: A case study of the haitian earthquake. World Medical \& Health Policy, 2:7-33. 